\title{
iRHOM2 is a critical pathogenic mediator of inflammatory arthritis
}

\author{
Priya Darshinee A. Issuree,, ${ }^{1,2}$ Thorsten Maretzky, ${ }^{1}$ David R. Mcllwain,,3,4,5 \\ Sébastien Monette, ${ }^{6}$ Xiaoping Qing, ${ }^{1}$ Philipp A. Lang, ${ }^{5}$ Steven L. Swendeman, ${ }^{1}$ \\ Kyung-Hyun Park-Min, ${ }^{1}$ Nikolaus Binder, ${ }^{1}$ George D. Kalliolias, ${ }^{1}$ Anna Yarilina, ${ }^{1}$ Keisuke Horiuchi, ${ }^{7}$ \\ Lionel B. Ivashkiv, ${ }^{1,2}$ Tak W. Mak, ${ }^{3,4}$ Jane E. Salmon, ${ }^{1,2,8}$ and Carl P. Blobel, ${ }^{1,8,9}$
}

\begin{abstract}
${ }^{1}$ Arthritis and Tissue Degeneration Program, Autoimmunity and Inflammation Program, Hospital for Special Surgery, and 2Department of Immunology, Weill Cornell University, New York, New York, USA. ${ }^{3}$ Campbell Family Institute for Breast Cancer Research, Ontario Cancer Institute, University Health Network, Toronto, Ontario, Canada. ${ }^{4}$ Department of Medical Biophysics, University of Toronto, Toronto, Ontario, Canada. ${ }^{5}$ Department of Gastroenterology, Hepatology, and Infectious Diseases, Heinrich-Heine University, Düsseldorf, Germany.

${ }^{6}$ Tri-Institutional Laboratory of Comparative Pathology, New York, New York, USA. ${ }^{7}$ Department of Orthopedic Surgery, School of Medicine, Keio University,

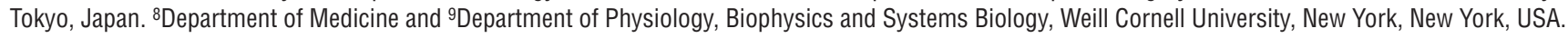

\begin{abstract}
iRHOM2, encoded by the gene Rbbdf2, regulates the maturation of the TNF- $\alpha$ convertase (TACE), which controls shedding of TNF- $\alpha$ and its biological activity in vivo. TACE is a potential target to treat TNF- $\alpha$-dependent diseases, such as rheumatoid arthritis, but there are concerns about potential side effects, because TACE also protects the skin and intestinal barrier by activating EGFR signaling. Here we report that inactivation of $R b b d f 2$ allows tissue-specific regulation of TACE by selectively preventing its maturation in immune cells, without affecting its homeostatic functions in other tissues. The related iRHOM1, which is widely expressed, except in hematopoietic cells, supported TACE maturation and shedding of the EGFR ligand TGF- $\alpha$ in Rbbdf2-deficient cells. Remarkably, mice lacking $R b b d f 2$ were protected from $\mathrm{K} / \mathrm{BxN}$ inflammatory arthritis to the same extent as mice lacking TACE in myeloid cells or Tnfa-deficient mice. In probing the underlying mechanism, we found that two main drivers of $\mathrm{K} / \mathrm{BxN}$ arthritis, complement $\mathrm{C} 5 \mathrm{a}$ and immune complexes, stimulated iRHOM2/ TACE-dependent shedding of TNF- $\alpha$ in mouse and human cells. These data demonstrate that iRHOM2 and myeloid-expressed TACE play a critical role in inflammatory arthritis and indicate that iRHOM2 is a potential therapeutic target for selective inactivation of TACE in myeloid cells.
\end{abstract}

\section{Introduction}

iRHOM2, an inactive member of the Rhomboid intramembrane proteinase family, was recently identified as regulator of the TNF- $\alpha$ convertase (TACE) (1-3). TACE is essential for activating the EGFR and for releasing TNF- $\alpha$ (4-8). Since biologic TNF- $\alpha$ blockers are widely used to treat rheumatoid arthritis (RA), a destructive, inflammatory joint disease affecting $0.5 \%-1 \%$ of the population, TACE is a potential alternative target for treatment of RA. However, a recently identified TACE-deficient patient revealed key roles for TACE in protecting the skin and intestinal barrier in humans (9). Notably, similar skin defects are recapitulated in mice lacking either TACE or EGFR in keratinocytes (10), and mice with strongly reduced TACE are susceptible to DSS colitis, a model for inflammatory bowel disease, probably because of a lack of EGFR signaling $(11,12)$. The protective role of TACE in the skin and intestine raises concerns about the potential side effects of targeting of TACE. It would be preferable to selectively inactivate TACE without compromising its protective functions, but, to date, there is no suitable approach to accomplish this. Here, we report that RHOM2 is a good target to inactivate TACE in myeloid cells without affecting its protective functions and that iRHOM2 might therefore be an attractive new target for treatment of RA.

\section{Results and Discussion}

iRHOM2 controls the maturation of TACE, yet $R b b d f 2^{-/-}$mice are healthy (1-3), whereas Tace $e^{-/}$mice die perinatally $(5,7)$. To

Authorship note: Jane E. Salmon and Carl P. Blobel contributed equally to this work. Conflict of interest: The authors have declared that no conflict of interest exists. Citation for this article: J Clin Invest. 2013;123(2):928-932. doi:10.1172/JCI66168. address this apparent paradox, we assessed whether iRHOM2 affects TACE maturation in tissues other than macrophages. In Western blots of Rhbdf2-/- tissues, mature TACE was not detected in bone marrow and was strongly reduced in lymph nodes but was clearly present in the brain, heart, kidney, liver, lung, and spleen (Figure 1A), in approximate concordance with the expression of the related iRHOM1 (BioGPS atlas, mu-iRHOM1). We therefore tested whether it is iRHOM1 that supports TACE maturation in Rhbdf2 $2^{-/}$mouse embryonic fibroblasts (mEFs), which expressed higher iRHOM1 levels than macrophages (Figure 1B and Supplemental Figure 1; supplemental material available online with this article; doi:10.1172/JCI66168DS1) and had normal levels of mature TACE in Western blots (Figure 1C; control: $\mathrm{Tace}^{-/-} \mathrm{mEFs}$ ). Rbbdf2 $-1-$ mEFs shed the TACE substrate and EGFR ligand, TGF- $\alpha$, at comparable levels to those of WT controls (Figure 1D). However, TGF- $\alpha$ shedding was strongly reduced in Rhbdf2- $2^{-/}$mEFs treated with iRhom1 siRNA (Figure 1D; control: $\mathrm{Tace}^{-/-} \mathrm{mEFs}$ ). Western blots showed normal mature TACE levels in iRhom1 siRNAtreated WT mEFs but strongly reduced mature TACE levels in iRhom 1 siRNA-treated Rhbdf2-/- mEFs; iRhom 1 siRNA was effective in both WT and mutant cells (Figure 1, E and F). Since iRHOM1 was not upregulated in Rbbdf2 $2^{-/}$mEFs (Figure 1B and Supplemental Figure 1), iRHOM1 was sufficient for TACE maturation and function. In Rbbdf2-- primary keratinocytes, which expressed similar iRHOM1 levels as mEFs (Figure 1B and Supplemental Figure 1), mature TACE levels and the release of endogenous TGF- $\alpha$ were comparable to those in controls (Figure 1, G and H). Since TACE and TGF are crucial for skin barrier maintenance in mice (10) and TACE/EGFR signaling protects from DSS colitis 
A
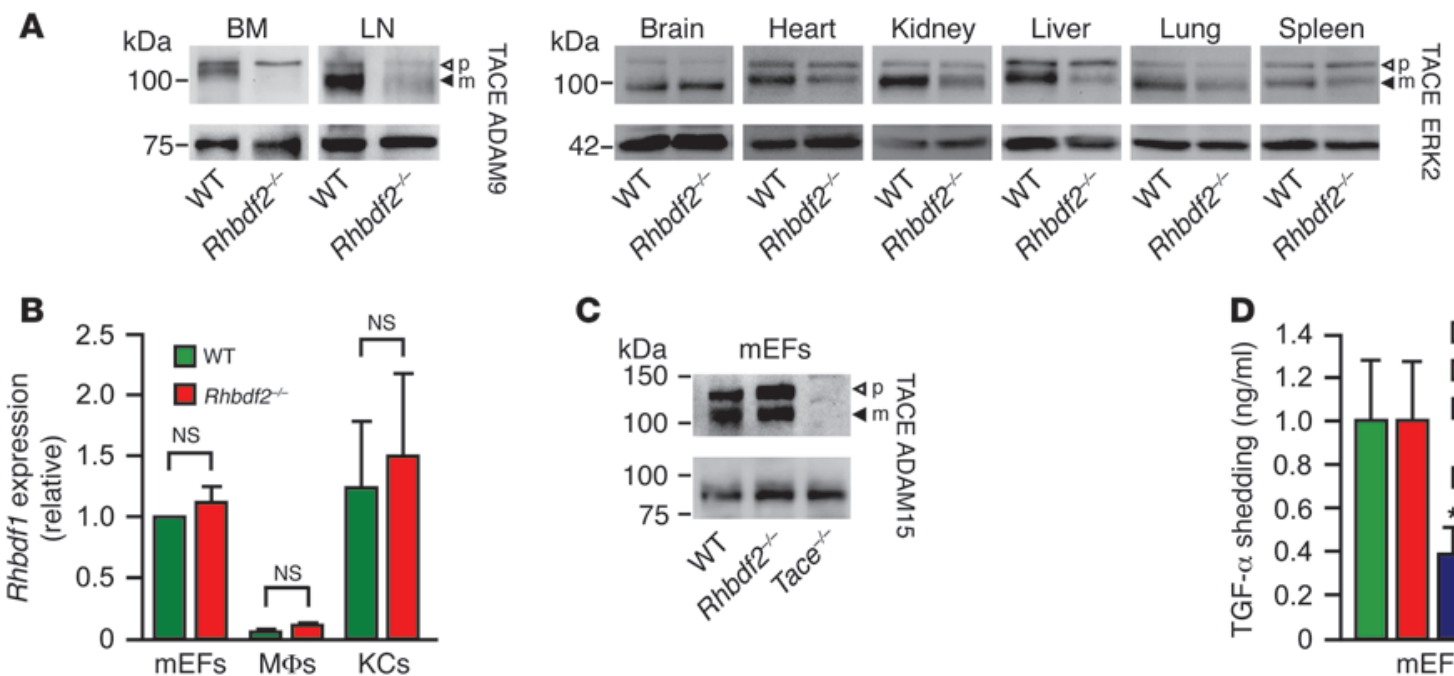

C
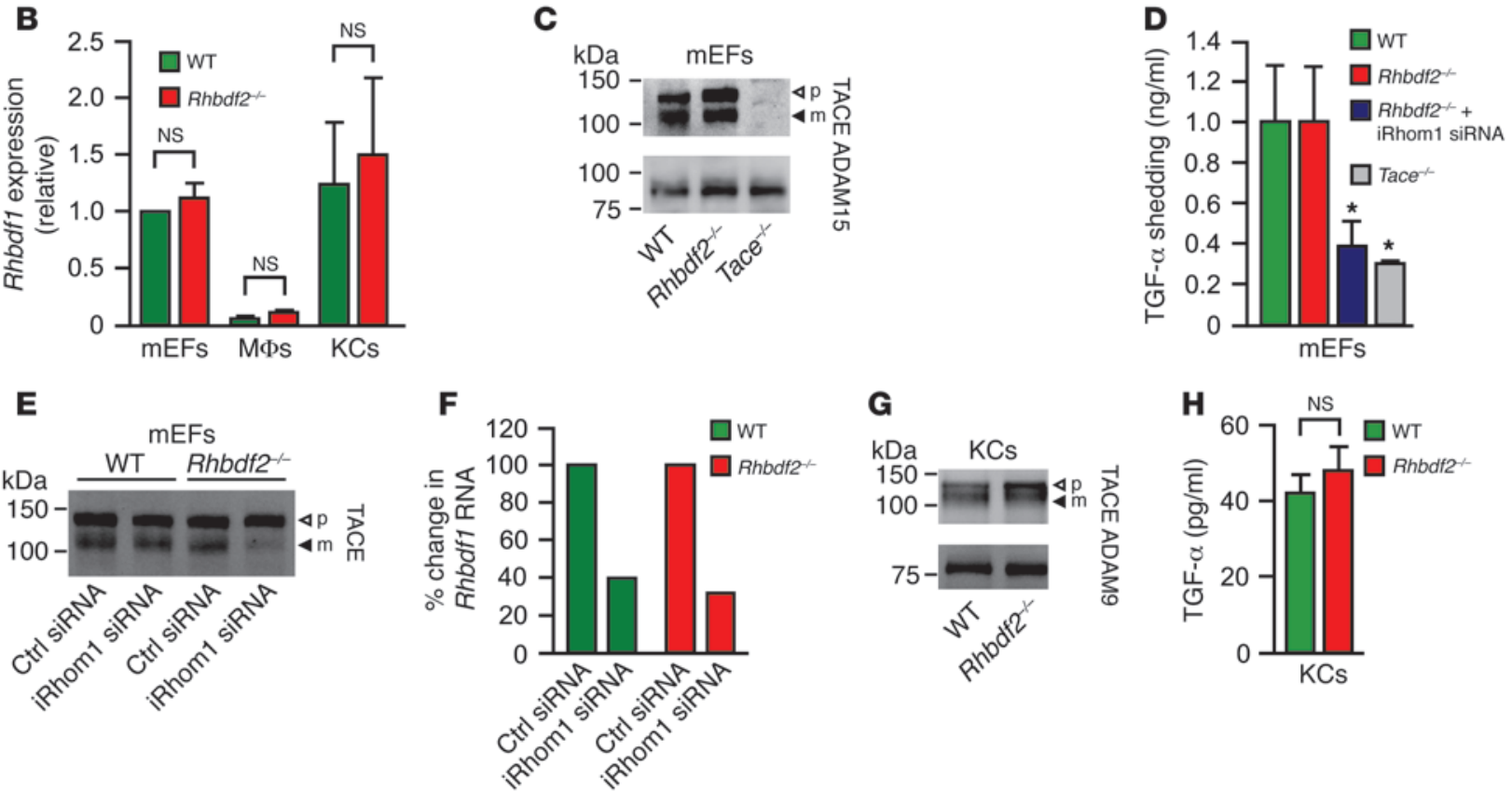

\section{Figure 1}

iRHOM2 controls TACE maturation in immune cells but not somatic tissues. (A) Western blots of TACE in tissues and cells from Rhbdf2-l- mice and littermate controls (WT). In Rhbdf2-/- mice, mature TACE is absent in bone marrow and strongly reduced in lymph nodes but present in brain, heart, kidney, liver, lung, and spleen (differences in mature TACE migration are caused by N-linked carbohydrate modifications; blots are representative of 5 experiments). p, pro-TACE; m, mature TACE. (B) qPCR of Rhbdf1 in mEFs, primary macrophages (MФs), and primary

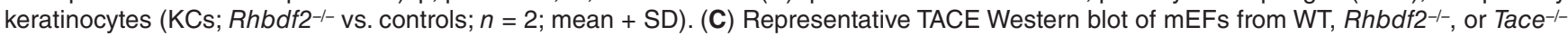

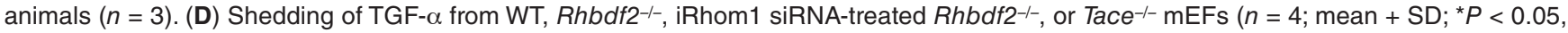
WT vs. iRhom1 siRNA-treated Rhbdf2-/- mEFs or WT vs. Tace ${ }^{-/-}$mEFs, 1-way ANOVA followed by Dunnett's test). (E) TACE Western blot shows reduction of mature TACE only in iRhom 1 siRNA-treated Rhbdf2-- mEFs but not in iRhom1 siRNA-treated WT controls (Ctrl). (F) qPCR confirmed reduction of Rhbdf1 in iRhom 1 siRNA-treated WT or Rhbdf2-/- mEFs (representative of 3 experiments). (G) Western blot of TACE and (H) release of endogenous TGF- $\alpha$ from primary keratinocytes from $R h b d f 2^{-/-}$or WT mice $(n=2$; mean + SD). ADAM9, ADAM15, or ERK2 were used as loading controls, as indicated.

$(11,12)$, these findings provide a compelling explanation for the lack of skin and intestinal defects in Rbbdf2-/- mice (3).

To explore the role of iRHOM2 in RA, we isolated synovial macrophages from patients with RA and found a significant upregulation of $\mathrm{iRHOM} 2$ expression compared with that in healthy controls (Figure 2A). We then assessed the role of iRHOM2 in the $\mathrm{K} / \mathrm{BxN}$ mouse RA model, which has a substantial TNF- $\alpha-$ dependent component (although some $\mathrm{Tnfa}^{-/-}$mice are not protected from disease; refs. 13, 14). $\mathrm{K} / \mathrm{BxN}$ inflammatory arthritis

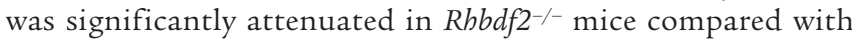
that in controls, showing less joint swelling (Figure 2B) and lower clinical scores (Supplemental Figure 2A), reflecting less synovial inflammation and cartilage erosion (Figure 2, C and D). These results show for what we believe to be the first time that iRHOM2 promotes inflammatory arthritis in the $\mathrm{K} / \mathrm{BxN}$ model. Since this model depends on innate immune cells, such as macrophages and neutrophils (15), and since Rbbdf2-/- mice have little, if any, active
TACE in these cells (Figure 2E and refs. 1, 2), we tested whether myeloid-specific inactivation of TACE would protect from $\mathrm{K} / \mathrm{BxN}$ arthritis. As in the case of Rhbdf2-/- mice (Figure 2, F-H, and Supplemental Figure $2 \mathrm{~B}$ ) or $\mathrm{Tnfa}^{-/-}$mice $(13,14)$, Tace $^{\Delta M C}$ mice (mice with Tace deleted $[\Delta]$ in myeloid cells) (5) were similarly protected from serum transfer arthritis. Thus, the protection of Rhbdf2-/mice from $\mathrm{K} / \mathrm{BxN}$ inflammatory arthritis most likely involves inactivation of TACE-dependent TNF- $\alpha$ release from myeloid cells.

To understand why Rhbdf2-/- and Tace ${ }^{\Delta M C}$ mice are protected from $\mathrm{K} / \mathrm{BxN}$ arthritis, we tested whether two crucial drivers of this model, C5a receptor and Fc $\gamma \mathrm{R}(13,15)$, activate iRHOM2/ TACE-dependent TNF- $\alpha$ shedding. We found that stimulation of Tace $^{\triangle M C}$ macrophages or Tace ${ }^{-/-}$fetal liver macrophages with C5a triggered markedly reduced TNF- $\alpha$ release compared with that in controls (Figure 3A). Human monocytes behaved similarly, in that C5 $a$ also activated TACE to shed TNF- $\alpha$, as evidenced by the inhibitory effect of TACE-selective hydroxamates (SP26 and DPC333; 
A

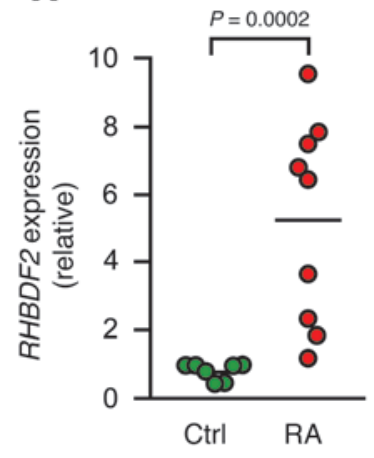

E

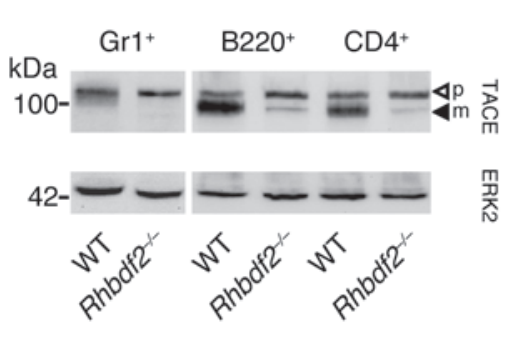

Figure 2

iRHOM2 and TACE in myeloid cells are key players in RA. (A) Relative RHBDF2 expression in RA synovial macrophages ( $n=9$ ) compared with that in macrophages from normal control patients $(n=7)$. The horizontal line indicates the mean. (B) Ankle thickness of Rhbdf2-l- $(n=11)$ and control $(n=14)$ mice injected with K/BxN serum (mean + SD). (C) Histology of ankle joints (left) and cartilage erosion (right) in Rhbdf2-/- and control mice (arrows indicate synovitis). Scale bars: $500 \mu \mathrm{m}$ (left); $20 \mu \mathrm{m}$ (right). (D) Blinded scoring of ankle joints from Rhbdf2--- mice ( $n=11$ ) or controls $(n=14)$ for inflammation and cartilage erosion (scale of 0 to 4$)$ (mean + SD). (E) Western blot of TACE on Gr1+ myeloid cells, B220 ${ }^{+}$B cells, and CD4+ $\mathrm{T}$ cells (ERK2 was used as a loading control) $(n=2)$. (F) Ankle swelling in Tace ${ }^{\triangle M C}$ and control mice subjected to K/BxN arthritis $(n=14$ each; mean + SD). (G) Histology (as in $\mathbf{C})$ and $(\mathbf{H})$ blinded scoring of ankle sections from Tace ${ }^{\Delta M C}$ mice and controls for inflammation and cartilage erosion $(n=14$ each; mean $+\mathrm{SD}) .{ }^{*} P<0.05$, Mann-Whitney $U$ test. ref. 16) and the TACE-specific inhibitory antibody D1(A12) (ref. 17 and Figure 3B). Moreover, crosslinking Fc $\gamma$ RIIa or Fc $\gamma$ RIII on human monocytes with mAbs or immune complexes (ICs) (18) stimulated TNF- $\alpha$ shedding, which was enhanced by C5a, and inhibited by DPC333 (Figure 3C). Treatment of human macrophages with $\mathrm{C} 5 \mathrm{a}$ or IC stimulated the production of RHBDF2 mRNA and TACE (Supplemental Figure 3). Addition of the TACE inhibitor DPC333 or etanercept reduced TNFA mRNA, suggesting that iRHOM2/TACE inhibitors could have unanticipated antiinflammatory benefits by reducing not only TNF- $\alpha$ shedding, but also its biosynthesis (Supplemental Figure 4). Of note, TACE was not required for other $\mathrm{C} 5 \mathrm{a}$ receptor-mediated and $\mathrm{Fc} \gamma$ receptormediated (C5aR-mediated and $\mathrm{F} c \gamma \mathrm{R}$-mediated) effector functions that are essential for microbial host defense, such as phagocytosis and generation of reactive oxidants (Supplemental Figure 5).

To determine whether iRHOM2 regulates TACE in human monocytes, these were treated with iRHOM2 siRNA, which reduced mature TACE, but not pro-TACE, as in Rhbdf2-/- BMderived macrophages (BMDMs) (1-3), whereas TACE siRNA reduced both forms (Figure 3, D and E). iRHOM2 siRNA or TACE siRNA significantly decreased the C5a-, IC-, or C5a/IC-stimu- c
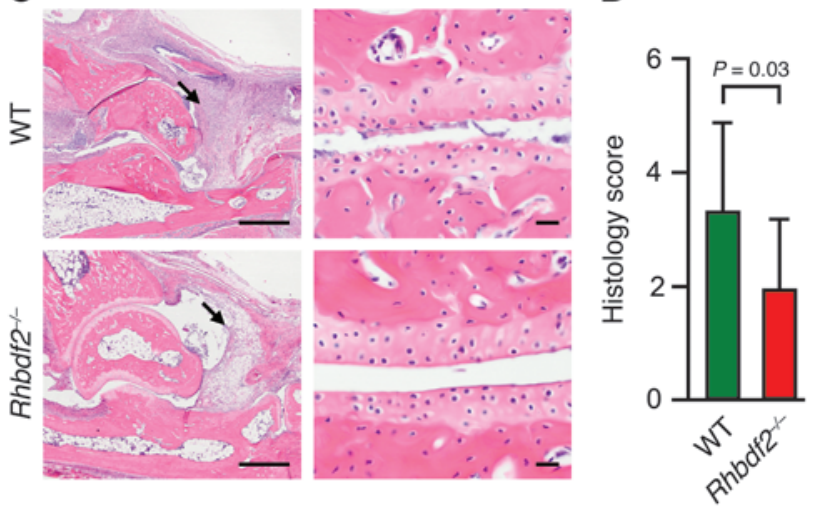

G
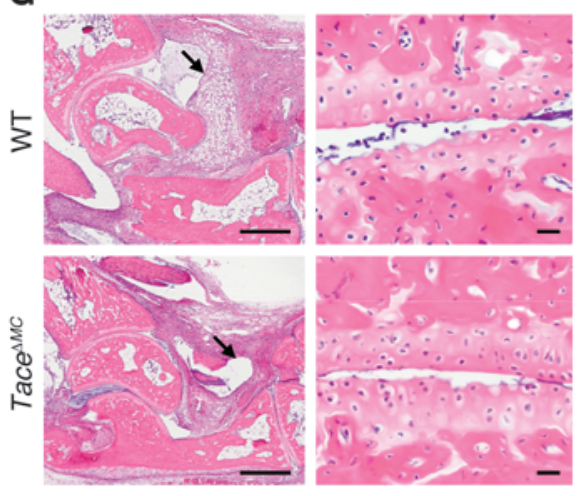

H

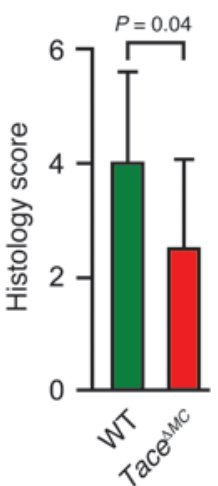

lated release of TNF- $\alpha$, establishing iRHOM2 as a key molecule in complement- and IC-stimulated TNF- $\alpha$ release from human monocytes (Figure 3F). C5a- and IC-stimulated TNF- $\alpha$ shedding were attenuated, in some cases differentially, by inhibitors of Src family kinases, p38MAPK, PKC, MEK, and Syk, some of which prevent arthritis in rodent models (Supplemental Figure 6). Finally, the in vivo relevance of TACE and iRHOM2 in C5aand IC-stimulated TNF- $\alpha$ release was confirmed in reverse passive Arthus reaction peritonitis (19), where soluble TNF- $\alpha$ was markedly reduced in the peritoneal lavage in Tace ${ }^{\Delta M C}$ and Rhbdf2-/mice (Figure 3G). Taken together, our results demonstrate for the first time to our knowledge that $\mathrm{C} 5 \mathrm{a}-\mathrm{C} 5 \mathrm{aR}$ and IC-Fc $\gamma \mathrm{R}$ both activate iRHOM2/TACE to release TNF- $\alpha$, providing mechanistic insights into how these 2 pathways promote inflammatory RA. The pathway by which C5a and ICs activate TACE is presumably sequential, as both stimuli can activate TACE strongly, but blocking either prevents serum transfer RA $(13,15)$.

In summary, our results explain why $R h b d f 2^{-/-}$mice display no obvious spontaneous pathologies: mature TACE is produced in most somatic tissues of Rhbdf2-/- mice. The related iRHOM1, which is expressed in somatic tissues but not in most hematopoi- 

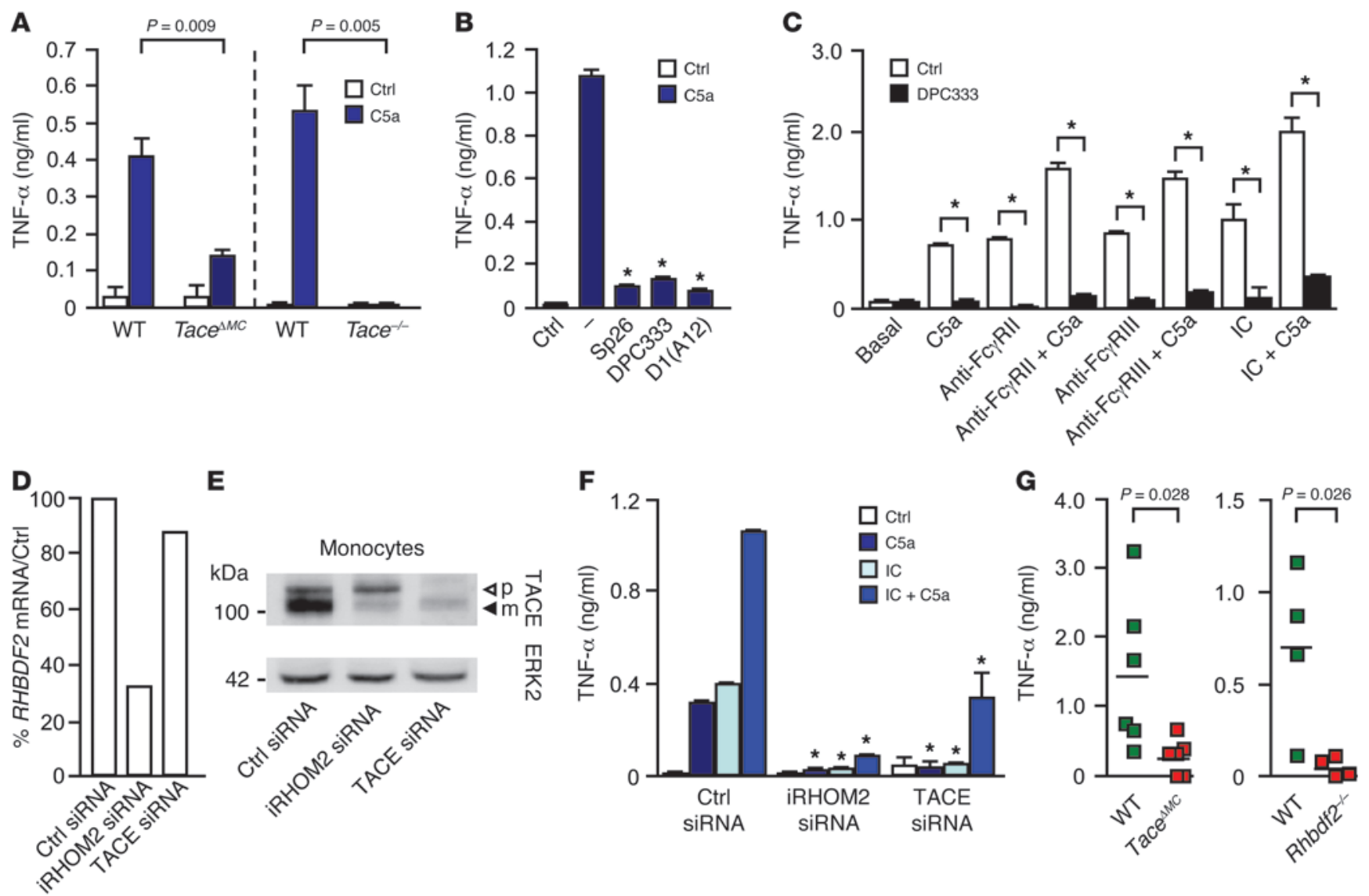

Figure 3

Complement C5a and ICs activate TACE to shed TNF- $\alpha$. (A) C5a-induced TNF- $\alpha$ shedding from Tace ${ }^{\triangle M C}$ BMDMs or fetal liver-derived macrophages from Tace ${ }^{-/-}$mice or controls, assessed by ELISA after 3 to 4 hours of stimulation $(n=4$; mean + SD). (B) TNF- $\alpha$ shedding by human monocytes during 2-hour stimulation with C5a $(1 \mu \mathrm{g} / \mathrm{ml})$ plus TACE inhibitors (SP26, DPC333) and anti-TACE antibody D1(A12) (representative of 4 experiments; mean $\left.+\mathrm{SD} ;{ }^{*} P<0.05\right)$. (C) TNF- $\alpha$ shedding by human monocytes induced by Fc $\gamma$ R crosslinking (mAbs or model IC [concentration, $0.25 \mathrm{mg} / \mathrm{ml} \mathrm{IVIg])} \mathrm{and/or} \mathrm{C5a}(1 \mu \mathrm{g} / \mathrm{ml})$, assessed after 2 hours (representative of 3 experiments; mean $+\mathrm{SD}$, ${ }^{\star} P<0.05$, DPC333 vs. control). (D) Human monocytes have reduced RHBDF2 mRNA when treated with iRHOM2 siRNA, but not with TACE siRNA, and (E) iRHOM2 siRNA treatment of human monocytes reduced mature TACE, but not pro-TACE, in a Western blot, whereas TACE siRNA reduced pro- and mature TACE $(n=3)$. (F) TNF- $\alpha$ release from C5a-, IC-, and IC/C5a-stimulated cells treated with or without iRHOM2 siRNA or TACE siRNA ( $n=3$; mean $+\mathrm{SD} ;{ }^{*} P<0.05$, stimulated control siRNA vs. iRHOM2 siRNA or TACE siRNA, 1-way ANOVA, followed by Dunnett's test). (G) Soluble TNF- $\alpha$ in the peritoneal lavage of $\operatorname{Tace}^{\Delta M C}(n=6)$ or $\operatorname{Rhbdf2}^{-/-}$mice $(n=4)$ and controls injected with ova plus anti-ova for 3 to 4 hours $(n=6)$.

etic cells, appears to support TACE maturation and function in the absence of $\mathrm{R}$ HOM2, as shown in fibroblasts. Importantly, the lack of mature TACE in immune cells of Rhbdf2-/- mice offers significant protection from inflammatory arthritis, comparable to what is observed in Tace ${ }^{\Delta M C}$ mice or $\mathrm{Tnfa}^{-/-}$mice. Because iRHOM2 and TACE are essential for the release of TNF- $\alpha$ from myeloid cells, and because TNF- $\alpha$ is a successful target for the treatment of RA, iRHOM2 may offer the unique and exciting prospect of cell type-specific blockade of TACE and TNF- $\alpha$ shedding. The skin and intestinal inflammation in a patient lacking TACE (9) emphasize the important opportunity provided by targeting iRHOM2 to limit immune cell-specific TACE activation in RA and possibly other TNF- $\alpha$-dependent pathologies, yet avoid the potential consequences of systemic TACE inactivation.

\section{Methods}

Mice. Tace ${ }^{\Delta M C}$, Tace ${ }^{-/-}$, and Rhbdf2 $2^{-/-}$mice $(2,5)$ were of $129 \mathrm{~Sv} / \mathrm{C} 57 \mathrm{BL} / 6$ mixed background. C5aR $\mathrm{R}^{-/-}$mice $(\mathrm{C} 57 \mathrm{BL} / 6)$ were from C. Gerard (20).
KRN-TCR mice (from D. Mathis and C. Benoist, Harvard Medical School, Boston, Massachusetts, USA) were mated with NOD/SHiLtJ mice (The Jackson Laboratory) to generate $\mathrm{K} / \mathrm{BxN}$ mice.

Reagents. Recombinant human C5a and C3a (EMD4Biosciences) and recombinant human TNF- $\alpha$ (R\&D Systems) were endotoxin free (Limulus Amebocyte Lysate Pyrochrome Kit, Cape Cod Inc.). E. coli LPS (Chemicon); SP26 (16) (Schering Plough); anti-TACE D1(A12) (17) (from G. Murphy and C. Tape, Cambridge University, Cambridge, United Kingdom); DPC333 (16) (from R. Waltermire, Brystol-Myers Squibb); FACS antibodies (BD Pharmingen); TGF- $\alpha$ ELISA (R\&D Systems); anti-iRHOM1 (Sigma-Aldrich); anti-TACE, anti-ADAM9, or anti-ADAM15 (Blobel lab); and signaling inhibitors (Milipore) were also used.

Cell culture. $\mathrm{CD} 14^{+}$monocytes were purified as described previously (21). Macrophages were derived by culturing monocytes for 16 to 24 hours in 10\% FBS $\alpha$-MEM (Invitrogen) and $10 \mathrm{ng} / \mathrm{ml} \mathrm{hM-CSF}$ (Peprotech). Mouse BMDMs (21) were maintained in DMEM, 20\% FBS, and $10 \mathrm{ng} / \mathrm{ml} \mathrm{mM-CSF}$. Keratinocytes and mEFs were isolated as described previously $(2,22)$. 
qPCR. Total RNA (RNeasy) was reverse transcribed (Oligo-dT/SuperScript RT III, Qiagen). qPCR (SYBR Green, ABI PRISM 7900HT; Applied Biosystems) was normalized to actin.

Western blots. Western blots were performed as described previously (2). Please see Supplemental Figure 7 for details and quantification.

RNA interference. TACE, iRhom2, or control siRNAs were used for transfection (Lonza Nucleofector). iRhom1 siRNA duplex (Rhbdf1-MSS203813, Rhbdf1-MSS203814, or Rhbdf1-MSS203815; Invitrogen) was also used for transfection (Lipofectamine 2000, Invitrogen). Data shown are representative for Rhbdf1-MSS203814 (see Figure 1, D-F). iRhom2 siRNA duplex (HSS128595, HSS188104, HSS128594; Invitrogen) was used for transfection. Data shown are representative for HSS188104 (see Figure 2, D-F).

Shedding assay. Shedding assays were for 3 to 4 hours (16).

Fc crosslinking. CD $14^{+}$mononuclear cells were pretreated with or without DPC333 and added to 96-well plates coated with anti-FcyRIII (3G8, Medarex), anti-FcrRII (IV.3, Medarex), or IVIG (Talecris Biotherapeutics).

Reverse passive peritoneal Arthus reaction. Following i.v. injection of $20 \mathrm{mg} / \mathrm{kg}$ chicken egg albumin (Sigma-Aldrich), $800 \mu \mathrm{g}$ rabbit anti-chicken egg albumin IgG (MP Biomedicals) was i.p. injected. i.p. anti-OVA and i.v. PBS were used as controls. Mice were sacrificed after 3 hours for peritoneal lavage (3 $\mathrm{ml}$ PBS, $1 \%$ heparin).

$K / B x N$ serum transfer arthritis. $100 \mu \mathrm{l}$ of serum from 6- to 8-week-old $\mathrm{K} / \mathrm{BxN}$ mice was injected i.p. at days 0 and 2. Clinical index and ankle thickness were measured (13). Ankle joint sections were scored (blinded) on a scale of 0 to 4 for inflammation and erosion (23).

1. Adrain C, Zettl M, Christova Y, Taylor N, Freeman $\mathrm{M}$. Tumor necrosis factor signaling requires iRhom 2 to promote trafficking and activation of TACE. Science. 2012;335(6065):225-228.

2. McIlwain DR, et al. iRhom 2 regulation of TACE controls TNF-mediated protection against Listeria and responses to LPS. Science. 2012;335(6065):229-232.

3. Siggs OM, et al. iRhom 2 is required for the secretion of mouse TNFalpha. Blood. 2012; 119(24):5769-5771.

4. Black R, et al. A metalloprotease disintegrin that releases tumour-necrosis factor- $\alpha$ from cells. Nature. 1997;385(6618):729-733.

5 . Horiuchi $\mathrm{K}$, et al. Cutting edge: TNF- $\alpha$-converting enzyme (TACE/ADAM17) inactivation in mouse myeloid cells prevents lethality from endotoxin shock. J Immunol. 2007;179(5):2686-2689.

6. Moss ML, et al. Cloning of a disintegrin metalloproteinase that processes precursor tumour-recrosis factor- $\alpha$. Nature. 1997;385(6618):733-736.

7. Peschon JJ, et al. An essential role for ectodomain shedding in mammalian development. Science. 1998; 282(5392):1281-1284.

8. Blobel CP. ADAMs: key players in EGFR-signaling, development and disease. Nat Rev Mol Cell Biol. $2005 ; 6: 32-43$.

9. Blaydon DC, et al. Inflammatory skin and bowel disease linked to ADAM17 deletion. NEnglJ Med.2011;

Synovial macrophages. Gene expression analysis was performed in RA synovial macrophages (deidentified; use of macrophages was approved by Hospital for Special Surgery IRB), which were isolated as described previously (24).

Statistics. An unpaired 2-tailed Student's $t$ test was used unless otherwise stated. SigmaStat3.1 software or Prism (GraphPad Software) was used for all statistical tests.

Study approval. All animal studies were approved by the Hospital for Special Surgery IACUC. All human studies were approved by the Hospital for Special Surgery IRB.

\section{Acknowledgments}

We thank G. Murphy and C. Tape for anti-huTACE antibodies and M. Vukelic, Y. Chinenov, E. Mogollon, A. Lee, and R. Gupte for technical assistance. Funding was provided by the NIH (R01 GM64750, to C.P. Blobel; AR38889, to J.E. Salmon; AR050401, to L.B. Ivashkiv; DFG LA2558/3-1, AvH SKA2010, to P.A. Lang; and AR061430 to K.-H. Park-Min).

Received for publication August 3, 2012, and accepted in revised form November 8, 2012.

Address correspondence to: Carl P. Blobel or Jane E. Salmon, Hospital for Special Surgery, 535 East 70th St., New York, New York 10021, USA. Phone: 212.606.1429; Fax: 212.774.2560; E-mail: blobelc@hss.edu (C.P. Blobel), salmonj@hss.edu (J.E. Salmon).

365(16):1502-1508.

10. Franzke CW, et al. Epidermal ADAM17 maintains the skin barrier by regulating EGFR ligand-dependent terminal keratinocyte differentiation. J Exp Med. 2012;209(6):1105-1119.

11. Chalaris A, et al. Critical role of the disintegrin metalloprotease ADAM17 for intestinal inflammation and regeneration in mice. J Exp Med. 2010; 207(8):1617-1624.

12. Brandl K, et al. MyD88 signaling in nonhematopoietic cells protects mice against induced colitis by regulating specific EGF receptor ligands. Proc Nat Acad Sci U S A. 2010;107(46):19967-19972.

13. Ji H, et al. Critical roles for interleukin 1 and tumor necrosis factor alpha in antibody-induced arthritis. J Exp Med. 2002;196(1):77-85.

14. Kitaura H, et al. Marrow stromal cells and osteoclast precursors differentially contribute to TNF-alpha-induced osteoclastogenesis in vivo. J Immunol. 2004;173(8):4838-4846.

15. Ji H, et al. Arthritis critically dependent on innate immune system players. Immunity. 2002; 16(2):157-168

16. Maretzky T, Zhou W, Huang XY, Blobel CP. A transforming Src mutant increases the bioavailability of EGFR ligands via stimulation of the cell-surface metalloproteinase ADAM17. Oncogene. 2011; 30(5):611-618.
17. Tape CJ, et al. Cross-domain inhibition of TACE ectodomain. Proc Natl Acad Sci U S A. 2011; 108(14):5578-5583.

18. Durandy A, et al. Intravenous immunoglobulins--understanding properties and mechanisms. Clin Exp Immunol. 2009;158(suppl 1):2-13.

19. Baumann $\mathrm{U}$, et al. A codominant role of $\mathrm{Fc}$ gamma $\mathrm{RI} / \mathrm{III}$ and $\mathrm{C} 5 \mathrm{aR}$ in the reverse Arthus reaction. J Immunol. 2000;164(2):1065-1070.

20. Hopken UE, Lu B, Gerard NP, Gerard C. Impaired inflammatory responses in the reverse arthus reaction through genetic deletion of the C5a receptor. J Exp Med. 1997;186(5):749-756.

21. Hu X, et al. Sensitization of IFN-gamma Jak-STAT signaling during macrophage activation. Nat Immunol. 2002;3(9):859-866.

22. Maretzky T, et al. Migration of growth factor-stimulated epithelial and endothelial cells depends on EGFR transactivation by ADAM17. Nat Commun. 2011;2:229.

23. Choe JY, Crain B, Wu SR, Corr M. Interleukin 1 receptor dependence of serum transferred arthritis can be circumvented by toll-like receptor 4 signaling. J Exp Med. 2003;197(4):537-542.

24. Antoniv TT, Ivashkiv LB. Dysregulation of interleukin-10-dependent gene expression in rheumatoid arthritis synovial macrophages. Arthritis Rheum. 2006;54(9):2711-2721. 